Martínez-García, I.; Padilla-Carmona, M.T. y Suárez-Ortega, M. (2019). Aplicación de la metodología Delphi a la identificación de factores de éxito en el emprendimiento. Revista de Investigación Educativa, 37(1), 129-146. DOI: http://dx.doi.org/10.6018/rie.37.1.320911

\title{
Aplicación de la metodología Delphi a la identificación de factores de éxito en el emprendimiento
}

\author{
Applying Delphi method to identification of success \\ factors in entrepreneurship*
}

\author{
Inmaculada Martínez-García, M. Teresa Padilla-Carmona, Magdalena Suárez-Ortega \\ Departamento Métodos de Investigación y Diagnóstico en Educación. Facultad de Ciencias de la Educación \\ Universidad de Sevilla (España)
}

\begin{abstract}
Resumen
A través de esta investigación se pretenden conocer los factores y competencias asociados al éxito en el desarrollo de un proyecto emprendedor tomando como referencia la experiencia de emprendedores consolidados que han llevado a cabo un proyecto empresarial. Para ello, se ha hecho uso de la metodología Delphi formada por cuatro fases en las que han participado 15 emprendedores consolidados procedentes de diferentes sectores laborales. Para la recogida de información se ha diseñado un cuestionario que ha derivado del proceso reiterativo y de las respuestas de los participantes, tal y como caracteriza a la metodología. Con este instrumento se ha recogido información sobre tres aspectos: factores que propician el éxito en el proyecto inicial de los emprendedores, competencias relacionadas con el mismo y estrategias a seguir por potenciales emprendedores. Los resultados muestran que los factores, competencias y recomendaciones que están relacionados con características y rasgos personales aparecen más vinculados al éxito del proyecto que aquellos relativos a los conocimientos o habilidades profesionales. La motivación para emprender, dedicación, ilusión, tiempo y esfuerzo son los factores percibidos como más relevantes para el éxito del proyecto emprendedor.

Palabras clave: metodología delphi; emprendimiento; desarrollo de la carrera; competencias; orientación profesional.

* La propuesta es resultado del proyecto titulado Diseño de la Carrera y Gestión del Talento Emprendedor (EDU2013-45704-P). Plan Estatal 2013-2016 Excelencia. Proyectos I+D. Ministerio de Economía y Competitividad. Correspondencia: Inmaculada Martínez García, inmamartinez@us.es, Departamento Métodos de Investigación y Diagnóstico en Educación, Facultad de Ciencias de la Educación, Universidad de Sevilla (España).
\end{abstract}




\begin{abstract}
This research aims to identify the factors and skills associated with success in the development of an entrepeneurial project based on the opinions of entrepreneurs with experience in carrying out a business project. Delphi method has been implemented throught four phases with 15 participants from different labor sectors. For data collection, in line with Delphi method, a questionnaire has been designed taking into account participants' answers along the reiterative process. Information has been collected on three basic aspects: factors that promote success in the initial project, skills related to it and strategies to be followed by potential entrepeneurs. The results show that the factors, competences and recommendations related to personal characteristics are more linked to the success of the project than those related to knowledge about the topic or professional skills. The motivation to start a project, dedication, enthusiasm, time and effort are perceived as the most relevant factors to the success of the entrepreneur project.

Keywords: delphi technique; entrepreneurship; career development; competences; career guidance.
\end{abstract}

\title{
Introducción
}

La crisis económica de la última década ha obligado a la población a adaptarse a las nuevas demandas del mercado laboral mediante la búsqueda de nuevos yacimientos de empleo como el autoempleo. Las últimas cifras establecen que, en España, existe un total de 1.542.725 de autónomos, 1.307.207 de PYMES y 1.135.211 microempresas (Ministerio de Economía, Industria y Competitividad, 2017). Esta actividad empresarial resulta clave en el crecimiento económico del país y es en este contexto donde el emprendimiento adquiere protagonismo. En este sentido, el Informe Mundial GEM (2017) pone énfasis en la relevancia del emprendimiento en la mejora y el aumento de la empleabilidad de un país, por este motivo surge el Global Entrepreneurship Monitor encargado del estudio de las dinámicas de emprendimiento a nivel internacional y del perfil de las personas que deciden emprender, a nivel individual.

El emprendimiento es entendido como proceso que comienza con la generación de una idea y continúa con su puesta en práctica y su lanzamiento al mercado. A este le seguirá la fase de consolidación tras un tiempo de vigencia (RED GEM, 2017). Tomando como referencia el Observatorio del Emprendimiento, la actividad emprendedora está vinculada al contexto social y cultural del país y a las actitudes y valores de su población. De esta forma, la actividad emprendedora en España (5.2\%) se desarrolla de manera muy diversa según la región, situándose en niveles inferiores a la media europea (8.4\%). Haciendo referencia al perfil de los emprendedores, el informe establece que el $26 \%$ lo hace por necesidad, al no percibir alternativas de empleo, siendo el $70.2 \%$ denominados emprendedores por oportunidad, partiendo de la detección de negocio en un área determinado. Asimismo, esta investigación pone en evidencia que en el contexto español existen condiciones favorables para emprender debido a los programas formativos y de seguimiento existentes. Sin embargo, 
hay carencias en la financiación (Messina \& Hochsztain, 2015) y en el desarrollo de iniciativas de sensibilización y fomento del emprendimiento desde etapas escolares tempranas (GEM, 2017).

En este contexto en el que las iniciativas empresariales emprendedoras cobran relevancia en el tejido productivo de un país, surge la necesidad de investigaciones que identifiquen factores y oportunidades de éxito en el emprendimiento (Davidsson, 2015; Devece, Peris-Ortiz \& Rueda-Armengot, 2016; Messina \& Hochsztain, 2015; Sastre, 2013; Wang, Ellinger \& Jim Wu, 2013). Un ejemplo es el de Porras, Oliveras y Vigier (2013), quienes hacen referencia a que, para que un proyecto empresarial sea exitoso "será imprescindible la concurrencia simultánea de dos elementos, una buena idea y un buen emprendedor" (p. 44), los autores enfatizan la necesidad del conocimiento del negocio a implementar y la formación específica en competencias emprendedoras.

La idea del emprendedor como protagonista y principal factor para el éxito del proyecto aparece de manera recurrente en la bibliografía reciente. Así, encontramos investigaciones que subrayan la relevancia de las características personales del emprendedor, como la honestidad, reputación, carisma y amabilidad (Partida, Carrera \& Villarreal, 2012). En esta misma línea, Sastre (2013) hace referencia a la relevancia de los factores intrínsecos del emprendedor a los que denomina virtudes (motivación, perseverancia, optimismo y confianza en uno mismo) como favorecedores del éxito del proyecto, sobre todo en las primeras etapas de creación de la empresa. Y también encontramos estudios que relacionan el liderazgo carismático y el concepto de emprendimiento (Van Hemmen, Urbano \& Álvarez, 2013). Un ejemplo es el de Varga (2010), quien establece que las personas con liderazgo emprendedor son percibidas con mayor probabilidad de éxito en la implementación de su negocio. Las características que se relacionan con este tipo de liderazgo son flexibilidad, mentalidad abierta al cambio, ser orientador de un grupo, contar con objetivos de logro, creatividad, potencial, toma de decisiones, solución de problemas, valores personales incluso se menciona el término "carisma" (Varga, 2010).

En este mismo sentido, autores como Pellicer (2014) hacen referencia a que existe cierto consenso en la bibliografía en percibir el emprender como una competencia a desarrollar a través del fomento de la "autonomía, creatividad, confianza, motivación de logro, liderazgo, eficacia y perseverancia" (p. 6). La autora también relaciona el emprendimiento con la disposición positiva hacia el cambio, motivaciones, planificación, constancia, saber hacer, trabajo en equipo, innovación, creatividad y búsqueda de soluciones, aspectos de carácter personal que son valorados como esenciales para conseguir el éxito en el proyecto emprendedor, siendo menos relevante en este proceso el modelo basado en conocimientos del sector o sobre el proceso de emprender. El JRC Science for Policy Report (2016) en su estudio presenta el EntreComp, un modelo conceptual que describe las que áreas que conforman la competencia emprendedora: ideas/oportunidades, recursos y acción. Este modelo pone en evidencia la relevancia de los aspectos personales identificados con el emprendimiento y que se relacionan con la búsqueda de oportunidades, creatividad, visión, valores, trabajo en equipo, 
aceptación de situaciones de riesgo, tomar la iniciativa, eficacia personal, motivación o perseverancia (Bacigalupo, Kampylis, Punie \& Van der Brande, 2016). Entender el emprendimiento como competencia la hace relevante para ser desarrollada en el contexto escolar de manera transversal a través de las etapas educativas y la creación de proyectos de educación emprendedora (Cárdenas \& Montoro, 2017), uno de los principales objetivos de la LOMCE (Ley Orgánica 8/2013, 2013).

Esta investigación se enmarca en un proyecto I+D+I en que se plantea la necesidad de diseñar estrategias formativas para el autoempleo basadas en la gestión de la carrera emprendedora. Para ello, en esta investigación, se analizan las percepciones de emprendedores consolidados sobre los factores que propician el éxito en el desarrollo de un proyecto emprendedor.

\section{Objetivos}

El objetivo general es estudiar los aspectos vinculados al éxito en la puesta en marcha de una iniciativa empresarial. Este nos lleva a formular los siguientes objetivos específicos:

- Identificar los factores personales y situacionales que influyen en la consolidación de un proyecto emprendedor.

- Analizar las competencias que se relacionan con el emprendimiento.

- Conocer las estrategias de éxito percibidas por emprendedores consolidados en el desarrollo de su proyecto empresarial.

\section{Método}

En esta investigación se ha hecho uso del método Delphi. El primer estudio que utiliza esta metodología es de Dalkey y Helmer (1962), quienes establecen que su objetivo es obtener el consenso a través de un grupo de expertos ante una temática concreta, usando para ello cuestionarios enviados individualmente de forma reiterada y cuyos resultados son devueltos en forma de retroalimentación creando una opinión representativa del grupo.

Sus numerosas ventajas han motivado la elección de esta metodología para esta investigación. Entre ellas destaca su flexibilidad al poder ser adaptada a muchas disciplinas científicas (Cabero \& Infante, 2014; López Gómez, 2018). Además de su versatilidad, Reguant-Álvarez y Torrado-Fonseca (2016) subrayan como positivo que las devoluciones de carácter controlado enviadas a los expertos con los resultados del grupo permiten la reflexión de los participantes. Presenta además otras ventajas como el carácter participativo en el proceso de construir significados entre expertos, contar con participantes con conocimientos y experiencia sobre la materia y, por último, el anonimato en las respuestas que evita que, ante un proceso de debate en grupo, existan personas que influencien las opiniones de los demás.

Su potencial es fundamentalmente prospectivo, es decir, genera información sobre la posible tendencia de un acontecimiento o las transformaciones futuras de un fenómeno. Por último, destacar que para su aplicación se hace uso de una metodología mixta con procedimientos de carácter cualitativo y cuantitativo, lo que enriquece el proceso de análisis de datos. 


\section{Participantes}

En la metodología Delphi la calidad de los resultados depende de la elección de los expertos participantes. Por ello, se contactó con 27 emprendedores experimentados, siendo 15 los que responden a la primera fase del estudio (Tabla 1). Se ha contado con una pérdida de sujetos a lo largo de las distintas fases prevista en este tipo de metodología, siendo 9 los participantes en la última fase ajustándose a las recomendaciones para los estudios de Delphi (Landeta, 2002).

Los informantes fueron seleccionados siguiendo tres criterios relevantes para la investigación: ser emprendedores consolidados, es decir, contar con un proyecto de emprendimiento en desarrollo; tener una trayectoria en emprendimiento y pertenecer a diferentes sectores empresariales. Contamos con representación del sector formativo/ educativo, diseño gráfico, servicios, agencias inmobiliarias y actividades profesionales científicas y técnicas.

Tabla 1

Participantes del estudio

\begin{tabular}{ccc}
\hline Fases & № Participantes & Sexo \\
\hline Fase 1 & 15 & \\
Fase 2 & 13 & Mujeres: 7 \\
Fase 3 & 13 & Hombres: 8 \\
Fase 4 & 9 & \\
\hline
\end{tabular}

\section{Fases en el desarrollo del método Delphi: procedimientos de recogida y análisis de la información}

Teniendo en cuenta las exigencias metodológicas y que un aspecto clave en esta metodología es la alternancia de un procedimiento cualitativo con otro más cuantificable, para el desarrollo de la investigación han sido diseñados cuatro instrumentos destinados a cada una de las fases de la recogida de datos (Figura 1). Su diseño ha estado determinado por la información aportada por los participantes en la fase previa tal y como la metodología lo exige. Los instrumentos han pasado de ser menos estructurados, formados por preguntas abiertas que no condicionan la dirección de las respuestas y que fomentan la generación de las ideas, a un nivel máximo de concreción en el que se solicitan ordenaciones, ponderaciones o preferencias con referentes numéricos, lo que permite realizar un tratamiento estadístico de la información que facilite la estimación del consenso.

Este diseño, basado en el mayor nivel de concreción en fases sucesivas, ha seguido unas pautas comunes reflejadas en los apartados que los componen, contando todas las plantillas elaboradas con un apartado introductorio de agradecimiento en el que se incluían las instrucciones de cumplimentación y estando divididas en tres blo- 
ques de contenido: factores que propician el éxito del proyecto empresarial, competencias relacionadas con el emprendimiento y estrategias a seguir por potenciales emprendedores.

El envío se ha hecho a través de correo electrónico indicando fecha de entrega y realizándose recordatorios para aquellos que no enviaron la plantilla cumplimentada en un primer momento.

La información ha sido analizada en función de la naturaleza de los datos recogidos, sin embargo, se debe tener en cuenta que el proceso ha sido inductivo y dependiente de los resultados de cada fase. Por tanto, tras la primera fase se realizó un análisis cualitativo de las respuestas de carácter abierto del que surge un sistema de categorías que es transformado en cuestionario para las fases posteriores. En relación a los datos de carácter cuantitativo se ha hecho uso de estadísticos descriptivos; frecuencias, medidas de tendencia central y valores de dispersión.

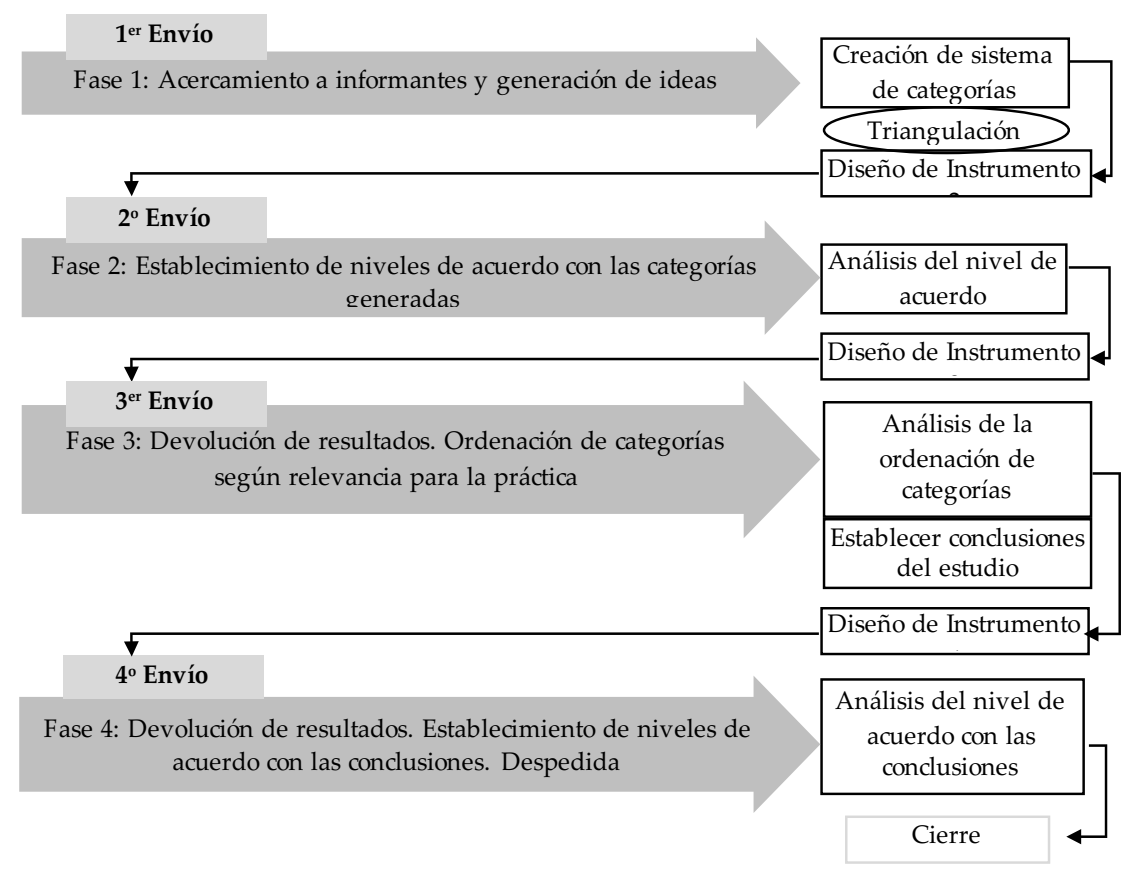

Figura 1. Fases del procedimiento y análisis de datos.

\section{Desarrollo y resultados por fases del estudio Delphi}

El método Delphi se caracteriza por su carácter reiterativo en el que se intercalan los procesos de recogida de información con el de análisis y devolución a los participantes, caracterizándose por estar en continua elaboración y por la retroalimentación ofrecida a los informantes en cada fase. Una vez ha sido alcanzado el consenso del grupo, se dan por finalizadas las rondas del mismo. Por tanto, en nuestro caso, el procedimiento de recogida de datos ha ido de la mano del análisis y presentación de resultados. Aten- 
diendo al grado de acuerdo con las respuestas otorgadas en cada una de las fases de recogida de datos, son presentados a continuación los resultados obtenidos derivados del análisis de las aportaciones de los participantes en cada una de ellas.

\section{Fase I: Primer acercamiento a los informantes y generación de ideas}

Esta primera fase es de acercamiento a las impresiones de los participantes sobre los factores de éxito de su proyecto empresarial, las competencias emprendedoras que se consideran necesarias para alcanzar dicho éxito y las recomendaciones a seguir por emprendedores potenciales. El cuestionario inicial presentado cuenta con tres preguntas abiertas a través de las que se indaga sobre estas temáticas (ver Tabla 2).

Tabla 2

Preguntas exploratorias

¿Cuáles han sido los factores que han propiciado el éxito de su proyecto inicial?

¿Qué competencias debe poseer la persona que emprende para tener éxito en su proyecto?

¿Qué recomendaciones y consejos daría a una persona que está en la fase inicial de desarrollo de su proyecto emprendedor y/o en el primer año de su puesta en marcha?

Tras la recogida de la información de las mismas, se realiza un análisis de las respuestas del que deriva un sistema de categorías para cada uno de los bloques temáticos presentados previamente. Con el objetivo de facilitar las fases posteriores, los bloques han sido conformados por 10 categorías cada uno de ellos, teniendo en cuenta no perder el sentido de las respuestas y el contenido de las mismas. Para ello, todas las opiniones emitidas son recogidas o bien en el título de la categoría o en los ejemplos propuestos en cada una de ellas.

Haciendo referencia a los factores que propician el éxito del proyecto emprendedor (Tabla 3) las categorías atienden a diferentes temáticas por las que pueden ser agrupadas. Por un lado, contamos con un grupo de categorías que están asociadas a factores de tipo personal o a la trayectoria previa, como pueden ser los aspectos motivacionales relacionados con el propio proyecto, aquellos vinculados a las propias competencias, los valores personales del profesional que pone en marcha el proyecto o su trayectoria formativa y experiencia previa. Por otro lado, los testimonios de los protagonistas hacen referencia a aquellos factores relacionados con la puesta en práctica del negocio como la planificación del mismo, la búsqueda del factor diferenciador del producto que es ofrecido, la inversión de tiempo y esfuerzo y los aspectos relacionados con los procesos de innovación y cambio. Por último, se mencionan aquellos factores vinculados a las relaciones sociales como la atención y relación con los clientes y la generación de alianzas y redes de apoyo. 
Tabla 3

Sistema de categorías factores de éxito del proyecto

\begin{tabular}{|c|c|}
\hline Categoría & Contenidos \\
\hline $\begin{array}{l}\text { Factores personales } \\
\text { vinculados a la } \\
\text { motivación en el propio } \\
\text { proyecto }\end{array}$ & $\begin{array}{l}\text { Ilusión, confianza en el proyecto, vocación, motivación y paciencia; } \\
\text { que te guste lo que haces; pasión. }\end{array}$ \\
\hline $\begin{array}{l}\text { Factores vinculados a las } \\
\text { competencias y valores } \\
\text { personales }\end{array}$ & $\begin{array}{l}\text { Competencias para desarrollar el trabajo, dotes comerciales; ca- } \\
\text { pacidad organizativa y de gestión; honradez, profesionalidad, } \\
\text { honestidad, sinceridad. }\end{array}$ \\
\hline $\begin{array}{l}\text { Factores vinculados a la } \\
\text { atención y relación con } \\
\text { los clientes }\end{array}$ & $\begin{array}{l}\text { Tener contactos, creación de una cartera de clientes; atención per- } \\
\text { sonalizada, cercanía y compromiso con el cliente. }\end{array}$ \\
\hline $\begin{array}{l}\text { Tener una buena } \\
\text { planificación comercial }\end{array}$ & Creación de una imagen corporativa (cuidada y con personalidad). \\
\hline $\begin{array}{l}\text { Variables relacionadas } \\
\text { con la planificación del } \\
\text { proyecto de negocio }\end{array}$ & $\begin{array}{l}\text { Definición de los servicios/productos ofrecidos (conocer sus po- } \\
\text { tencialidades y limitaciones); buena elección del nicho de mer- } \\
\text { cado, conocimiento del sector; planificación económica (contener } \\
\text { el gasto, saber dimensionar la estructura del proyecto y ajustarse). }\end{array}$ \\
\hline $\begin{array}{l}\text { Factor diferenciador del } \\
\text { producto }\end{array}$ & $\begin{array}{l}\text { Detectar necesidades que nadie satisface; creación de un producto } \\
\text { diferente; ofrecer servicios con mayor valor añadido; ofrecer un } \\
\text { sistema de distribución innovador y cómodo. }\end{array}$ \\
\hline $\begin{array}{l}\text { Aspectos relacionados } \\
\text { con procesos de } \\
\text { innovación y cambio }\end{array}$ & $\begin{array}{l}\text { Capacidad de adaptación a un entorno cambiante, nuevas nece- } \\
\text { sidades y situaciones; innovar y reinventarse; actualización y for- } \\
\text { mación continua. }\end{array}$ \\
\hline $\begin{array}{l}\text { Generación de alianzas y } \\
\text { redes de apoyo }\end{array}$ & $\begin{array}{l}\text { Contar con una red de contactos personales; rodearse de colab- } \\
\text { oradores (proactivos, con alta cualificación y con distintos per- } \\
\text { files); apoyo familiar; contar con apoyo docente. }\end{array}$ \\
\hline $\begin{array}{l}\text { Inversión de tiempo y } \\
\text { esfuerzo }\end{array}$ & $\begin{array}{l}\text { Dedicación personal; constancia y paciencia; tiempo invertido en } \\
\text { la empresa. }\end{array}$ \\
\hline $\begin{array}{l}\text { Trayectoria formativa } \\
\text { y/o experiencia } \\
\text { emprendedora previa }\end{array}$ & $\begin{array}{l}\text { Formación académica orientada al sector; formación previa; haber } \\
\text { emprendido en proyectos previos y diferentes. }\end{array}$ \\
\hline
\end{tabular}

Con relación a las competencias que los informantes asocian a su faceta como emprendedores y que propician el éxito de su proyecto (Tabla 4), las categorías creadas partiendo de los testimonios pueden ser agrupadas en aquellas más relacionadas con aspectos de tipo personal y aquellas vinculadas al proyecto empresarial. En el primer grupo nos encontramos competencias relacionadas con el conocimiento de uno mismo agrupadas bajo la categoría capacidad de trabajo, capacidad de aprendizaje continuo, resolución de problemas, competencias comunicativas y la capacidad de asumir fracasos y gestionarlos. Por otro lado, están aquellas competencias vinculadas a la puesta en marcha del proyecto empresarial, las competencias profesionales, competencias empresariales y comerciales, organizativas y de gestión del estrés, y la creatividad y capacidad de innovación. 
Tabla 4

Sistema de categorías competencias de la persona emprendedora para el éxito

\begin{tabular}{|c|c|}
\hline Categoría & Contenidos \\
\hline $\begin{array}{l}\text { Competencias relacionadas } \\
\text { con la capacidad de trabajo }\end{array}$ & $\begin{array}{l}\text { Dedicar muchas horas; constancia; tesón; paciencia; perseveran- } \\
\text { cia. }\end{array}$ \\
\hline $\begin{array}{l}\text { Competencias personales } \\
\text { y/o conocimiento de uno } \\
\text { mismo }\end{array}$ & $\begin{array}{l}\text { Contar con personalidad fuerte y autoconfianza; seguridad en } \\
\text { uno mismo; convencimiento personal; conocer las propias cuali- } \\
\text { dades y aptitudes y saber explotarlas. }\end{array}$ \\
\hline $\begin{array}{l}\text { Competencias relacionadas } \\
\text { con la capacidad de } \\
\text { resolución de problemas }\end{array}$ & $\begin{array}{l}\text { Ser resolutivo; manejo de situaciones críticas; resolución de con- } \\
\text { flictos. }\end{array}$ \\
\hline $\begin{array}{l}\text { Competencias } \\
\text { comunicativas y de trato } \\
\text { personal (o habilidades } \\
\text { sociales) }\end{array}$ & $\begin{array}{l}\text { Saber transmitir lo que se vende; capacidad de negociación; em- } \\
\text { patía; comunicación interpersonal; liderazgo y trabajo en equipo. }\end{array}$ \\
\hline $\begin{array}{l}\text { Competencias creativas y } \\
\text { capacidad de innovación }\end{array}$ & $\begin{array}{l}\text { Búsqueda de nuevas alternativas para la mejora y la excelencia; } \\
\text { saber adaptarse a los cambios/necesidades del entorno; voluntad } \\
\text { de investigar; capacidad de adaptación al mercado; renovarse. }\end{array}$ \\
\hline $\begin{array}{l}\text { Capacidad de aprendizaje } \\
\text { continuo }\end{array}$ & $\begin{array}{l}\text { Ganas de aprender y actualizarse constantemente; voluntad de } \\
\text { formación y mejora continua. }\end{array}$ \\
\hline $\begin{array}{l}\text { Competencias relacionadas } \\
\text { con aprender a asumir } \\
\text { fracasos y gestionarlos }\end{array}$ & $\begin{array}{l}\text { Resiliencia; maleabilidad (observación de fallos y virtudes para } \\
\text { aprender de ellos); ver los obstáculos como retos; soportar mo- } \\
\text { mentos de tensión; aceptar críticas del entorno; capacidad de } \\
\text { aprender de los errores y reveses. }\end{array}$ \\
\hline Competencias profesionales & $\begin{array}{l}\text { Actitud de profesionalidad; formación previa; conocimientos del } \\
\text { sector; formación adecuada al sector, manejo de nuevas tecnologías. }\end{array}$ \\
\hline $\begin{array}{l}\text { Competencias empresariales } \\
\text { y comerciales }\end{array}$ & $\begin{array}{l}\text { Conocimientos empresariales; gestión de recursos; conocimien- } \\
\text { tos fiscales, mercantiles y contables; competencias de marketing; } \\
\text { competencias en gestión de recursos humanos, dotes comerciales. }\end{array}$ \\
\hline $\begin{array}{l}\text { Competencias organizativas } \\
\text { y gestión del estrés }\end{array}$ & $\begin{array}{l}\text { Planificación; evaluación de riesgos y oportunidades; orga- } \\
\text { nización; toma de decisiones; fijación de metas; gestión del estrés. }\end{array}$ \\
\hline
\end{tabular}

Finalmente se presentan los resultados en torno a las recomendaciones y consejos de los emprendedores consolidados a las personas en sus inicios o en transición al emprendimiento (Tabla 5). Entre las categorías que fueron creadas, al igual que ocurría en los casos anteriores, nos encontramos tanto las más relacionadas con el ámbito personal como las que tienen que ver con el proceso de creación de la empresa. Entre las del primer grupo están las agrupadas bajo la etiqueta pedir ayuda/consejo, contar con un conjunto de cualidades personales, cuidar la formación continua, tener disponibilidad para invertir tiempo y dedicación personal, así como prestar atención a las relaciones sociales. Haciendo referencia a aquellas más relacionadas con el proyecto empresarial, los expertos mencionan la necesidad de hacer una adecuada planificación del proyecto, prestar atención a la publicidad, establecer networks con otros profesionales, cuidar la economía y apostar por la innovación y el cambio. 
Tabla 5

Sistema de categorías estrategias a seguir por potenciales emprendedores

\begin{tabular}{cl}
\hline \multicolumn{1}{c}{ Categoría } & \multicolumn{1}{c}{ Contenidos } \\
\hline Pedir ayuda/consejo & $\begin{array}{l}\text { Preguntar en el entorno próximo (clientes, colaboradores, expertos); } \\
\text { buscar asesoramiento, mentoría, de emprendedores de éxito y con } \\
\text { experiencia. }\end{array}$
\end{tabular}

Establecer networks

Establecer relaciones con otros profesionales en gestión y materia fiscal; seleccionar socios para colaboración y ayuda; definir funciones; crear un equipo multidisciplinar; hacer networking con otros emprendedores y empresas del ecosistema.

Hacer una adecuada planificación del proyecto empresarial

Prestar atención a la publicidad

Innovación y cambio

Cuidar la economía

Fijar objetivos ambiciosos pero realistas y factibles; definir el público objetivo; planificar, prever actuaciones; realizar análisis previo del producto; centrarse en un producto/servicio; ofrecer un producto que nadie ofrece; valorar sus fortalezas, debilidades, oportunidades y amenazas; contar con un estudio de mercado de viabilidad y económico; estudiar, conocer a la competencia; desarrollar un producto viable mínimo y validarlo con potenciales clientes; utilizar metodologías ágiles de diseño de negocio para mejorar el producto/ servicio antes de su lanzamiento.

Tener presencia en las redes sociales; invertir en marketing y publicidad; invertir en una imagen de marca; salir a vender, salir a venderse, buscar clientes; ser activos en las labores comerciales; atender las necesidades de publicidad.

Adaptar el producto a las necesidades surgidas; ir amoldando el producto/servicio a la realidad y necesidades cambiantes; tener claro que las ideas previas (sobre los clientes, sobre las necesidades de mercado que se pretende abrir y sobre la solución que se propone); apostar por las nuevas tecnologías e incorporarlas al negocio.

Tener un buen fondo de tesorería mientras llegan resultados; ser contenidos en el gasto (especialmente en trabajos y cobros estacionales); no "volverse loco" al principio; invertir beneficios en el negocio para crear crecimiento futuro; hacer un presupuesto de gasto realista.

Tener un conjunto de cualidades personales

No perder la motivación y la ilusión, tener paciencia perseverancia y constancia (especialmente en la fase inicial); mostrar fiabilidad profesional; no desesperar si al principio hay pocos resultados o no son rentables; afrontar los retos con valentía.

Prestar atención a las relaciones sociales

\section{Cuidar la formación continua}

Tener disponibilidad para invertir tiempo y dedicación personal
Perder el miedo y dominar las relaciones sociales; aprender a venderse; aprender a trabajar en equipo, delegar y escuchar las ideas de otros; seleccionar bien los socios y delimitar las funciones.

Formación en contabilidad analítica y financiera; formarse de forma continua, actualizar los conocimientos (el mercado, los clientes, ...).

Evaluación previa de tiempo/recursos personales y familiares; trabajo continuo y dedicación; revisar la escala de prioridades y saber qué sacrificios se está dispuesto a hacer. 


\section{Fase 2: Estimación del acuerdo inicial con las categorías generadas}

Antes de pasar a una clasificación de respuestas, como es habitual en el Delphi, comprobamos que la categorización realizada por las investigadoras recogía todas sus respuestas y el sentido de las mismas y si estas les parecían adecuadas para cada pregunta. De esta forma se podía identificar si existían factores que hubieran quedado fuera en la ronda anterior, a fin de incluirlos en una fase posterior. Por tanto, tomando como referencia el análisis de las respuestas obtenidas en la primera fase, esta información es enviada a los participantes. En el instrumento enviado se deja un espacio para que indiquen el grado de acuerdo general con la agrupación presentada, con valores porcentuales comprendidos entre 0, ningún acuerdo y 100 máximo acuerdo. El porcentaje de acuerdo mínimo exigido era del $70 \%$ por tanto, un menor nivel de acuerdo requería indicar la razón quedando un espacio para ello en el instrumento.

Tras ser recogidos los datos de la segunda fase, obtenemos un promedio de nivel de acuerdo general de $89.21 \%$ (89.3\% primer bloque; $90.8 \%$ segundo y $87.5 \%$ tercero), valores estimados como indicadores de un buen nivel de consenso y, por tanto, se lleva a cabo la tercera fase de recogida de datos.

\section{Fase 3: Devolución de los emprendedores y ordenación de las categorías según rele- vancia en la práctica}

Los datos relativos al nivel de acuerdo de la fase anterior son ofrecidos a los participantes en esta tercera fase junto al sistema de categorías, el objetivo es que ordenen los factores, competencias y recomendaciones correspondientes a cada uno de los bloques de contenido atendiendo al grado de importancia otorgado basándose en su experiencia. Para ello deben hacer uso de una escala numérica del 1 al 10, siendo 1 el factor, la competencia o recomendación más importante de la pregunta en cuestión, 2 el segundo más importante y así hasta el valor 10 que indicaría la variable menos relevante.

Haciendo referencia a los factores que propician el éxito del proyecto emprendedor, como se observa en los valores relativos al rango promedio de las puntuaciones otorgadas (Tabla 6), el denominado factores personales vinculados a la motivación en el propio proyecto es situado en las primeras posiciones como elemento principal que ha propiciado el éxito de su proyecto (media 2.54), seguido del factor diferenciador del producto (media 3.92) y de aquellos vinculados a las propias competencias y valores personales (media 4.15).

Por otra parte, como aspectos valorados como menos relevantes para la consecución de dicho éxito y situados en posiciones finales, nos encontramos con factores como la trayectoria formativa y/o experiencia emprendedora previa (media 7.62) como el menos relevante. Pero también no encontramos con los procesos de innovación y cambio (media 7.31) y la generación de alianzas y redes de apoyo (media 7.15) como elementos percibidos como menos relevantes para el éxito.

Las desviaciones típicas muestran que existe un alto nivel de acuerdo de los emprendedores consolidados en torno a establecer un orden de los factores que consideran que han propiciado el éxito en su proyecto de emprendimiento. En el mismo sentido, los datos obtenidos en relación al coeficiente de variación muestran que existe poca variabilidad en cuanto a la organización de los factores, lo que indica estabilidad en la respuesta y consenso en el momento de priorizar. 
Tabla 6

Factores que propician el éxito del proyecto emprendedor

\begin{tabular}{|c|c|c|c|c|}
\hline Factores de éxito & Posición & Rango promedio & DT & C. V. \\
\hline $\begin{array}{l}\text { Factores personales vinculados a la } \\
\text { motivación en el propio proyecto }\end{array}$ & 1 & 2.54 & 2.025 & 0.79 \\
\hline Factor diferenciador del producto & 2 & 3.92 & 2.362 & 0.60 \\
\hline $\begin{array}{l}\text { Factores vinculados a las competencias y } \\
\text { valores personales }\end{array}$ & 3 & 4.15 & 2.794 & 0.67 \\
\hline $\begin{array}{l}\text { Variables relacionadas con la planificación } \\
\text { del proyecto de negocio }\end{array}$ & 4 & 4.77 & 2.555 & 0.53 \\
\hline $\begin{array}{l}\text { Factores vinculados a la atención/relación } \\
\text { con los clientes }\end{array}$ & 5 & 5.08 & 2.871 & 0.56 \\
\hline Inversión de tiempo y esfuerzo & 6 & 5.15 & 2.075 & 0.40 \\
\hline Tener una buena planificación comercial & 7 & 7.08 & 2.139 & 0.30 \\
\hline Generación de alianzas y redes de apoyo & 8 & 7.15 & 1.994 & 0.27 \\
\hline $\begin{array}{l}\text { Aspectos relacionados con procesos de } \\
\text { innovación y cambio }\end{array}$ & 9 & 7.31 & 2.496 & 0.34 \\
\hline $\begin{array}{l}\text { Trayectoria formativa y/o experiencia } \\
\text { emprendedora previa }\end{array}$ & 10 & 7.62 & 2.725 & 0.35 \\
\hline
\end{tabular}

Haciendo referencia a la priorización que realizan de las competencias de la persona emprendedora, lo expertos a través de su posicionamiento (Tabla 7), sitúan a las competencias comunicativas y de trato personal en la primera posición (media 3.31) como competencias para el éxito de la persona emprendedora, seguidas de las competencias personales y/o conocimiento de uno mismo (media 3.77) y las competencias relacionadas con la capacidad de resolución de problemas (media 4).

Aquellas valoradas como menos relevantes, es decir, situadas en las posiciones finales son las competencias empresariales y comerciales (media 7.77), las vinculadas con la capacidad de asumir fracasos y gestionarlos (media 6.92) y las competencias organizativas y gestión del estrés (media 6.46).

Al igual que ocurría en el caso de los factores, los valores de la desviación típica y el coeficiente de variación muestran que existe un cierto nivel de acuerdo en la priorización haciéndose ver la estabilidad en la respuesta de los participantes y el alto nivel de consenso.

En relación a las estrategias a seguir por potenciales emprendedores, los expertos (Tabla 8) sitúan en primera posición aquellas relacionadas con una adecuada planificación del proyecto empresarial (media 3.38) seguidas de las cualidades y características personales a aprovechar (media 4.08) y del cuidado de la economía (media 4.69). Las recomendaciones valoradas como menos relevantes son el pedir ayuda y consejo (media 7.54), el cuidado de la formación continua (media 6.92) y los esfuerzos puestos en la publicidad del producto o servicio (media 6.15). 
Tabla 7

Competencias de la persona emprendedora para el éxito

\begin{tabular}{lcccc}
\hline \multicolumn{1}{c}{ Competencias clave en el emprendimiento } & Posición & Rango promedio & DT & C. V. \\
\hline $\begin{array}{l}\text { Competencias comunicativas y de trato personal } \\
\text { (o habilidades sociales) }\end{array}$ & 1 & 3.31 & 2.689 & 0.81 \\
$\begin{array}{l}\text { Competencias personales y/o conocimiento de } \\
\text { uno mismo }\end{array}$ & 2 & 3.77 & 2.713 & 0.71 \\
$\begin{array}{l}\text { Competencias relacionadas con la capacidad de } \\
\text { resolución de problemas }\end{array}$ & 3 & 4.00 & 1.915 & 0.47 \\
$\begin{array}{l}\text { Competencias relacionadas con la capacidad de } \\
\text { trabajo }\end{array}$ & 4 & 4.15 & 2.267 & 0.54 \\
$\begin{array}{l}\text { Competencias creativas e innovación } \\
\text { Capacidad de aprendizaje continuo }\end{array}$ & 5 & 5.31 & 2.594 & 0.48 \\
Competencias profesionales & 6 & 6.23 & 2.315 & 0.37 \\
Competencias organizativas y gestión del estrés & 8 & 6.31 & 3.276 & 0.51 \\
Competencias relacionadas con aprender a & 9 & 6.46 & 2.537 & 0.39 \\
asumir fracasos y gestionarlos & & 6.92 & 2.326 & 0.33 \\
Competencias empresariales y comerciales & 10 & 7.77 & 2.386 & 0.30 \\
\hline
\end{tabular}

En relación a los valores de la desviación típica y el coeficiente de variación, se puede observar que también existe un cierto nivel de acuerdo en la priorización en cuanto a esta variable. Si bien es cierto que existe cierta variabilidad en las opiniones en el caso del ítem hacer una adecuada planificación del proyecto empresarial, situado según la media en primera posición (media 3.38) pero contando con una desviación típica de 3.525 y un coeficiente de variación de 1.04, lo de denota una variabilidad en la respuesta mayor que en el resto de ítems donde encontramos más consenso en la priorización.

Tabla 8

Recomendaciones y consejos para las personas en la fase inicial

\begin{tabular}{lcccc}
\hline \multicolumn{1}{c}{$\begin{array}{c}\text { Recomendaciones y consejos para el } \\
\text { emprendimiento }\end{array}$} & Posición & Rango promedio & DT & C. V. \\
\hline $\begin{array}{l}\text { Planificación adecuada del proyecto } \\
\text { empresarial }\end{array}$ & 1 & 3.38 & 3.525 & 1.04 \\
Tener un conjunto de cualidades personales & 2 & 4.08 & 2.019 & 0.49 \\
Cuidar la economía & 3 & 4.69 & 2.097 & 0.44 \\
Establecer networks & 4 & 5.08 & 3.252 & 0.64 \\
$\begin{array}{l}\text { Disponibilidad para invertir tiempo y } \\
\text { dedicación personal }\end{array}$ & 5 & 5.23 & 3.563 & 0.68
\end{tabular}




\begin{tabular}{lcccc} 
Atender a las relaciones sociales & 6 & 6.08 & 2.499 & 0.41 \\
Innovación y cambio & 7 & 6.08 & 2.871 & 0.47 \\
Prestar atención a la publicidad & 8 & 6.15 & 2.410 & 0.39 \\
Cuidar la formación continua & 9 & 6.92 & 2.326 & 0.33 \\
Pedir ayuda/consejo & 10 & 7.54 & 2.025 & 0.26 \\
\hline
\end{tabular}

\section{Fase 4: Devolución y cierre}

A pesar de que todos los factores presentados han sido formulados por los participantes, en la priorización afloran factores y competencias que aluden a aspectos de carácter más personalista y que son situados de manera mayoritaria en las primeras posiciones. De esta forma, los resultados de la fase 3 muestran que los factores asociados al éxito por los participantes se encuentran más relacionados con aspectos y competencias de carácter personal en lugar de aquellos relacionados con el proceso de creación, desarrollo empresarial o las competencias propiamente comerciales. Este es el principal motivo por el que, pese al existir consenso general (Tablas 6, 7 y 8), quisimos añadir esta última fase con doble finalidad a) devolver a los participantes una síntesis de los resultados, y b) recabar de nuevo su aprobación general con las conclusiones obtenidas en la fase 3. Estas son agrupadas y presentadas a modo de resultados de las aportaciones de los informantes a lo largo de las fases previas. La presentación se realiza siguiendo los bloques de contenido iniciales y se pide a los participantes su nivel de acuerdo con las conclusiones derivadas del análisis previo.

Con el objeto de averiguar hasta qué punto es relevante esta priorización que pone el acento en un modelo de emprendedor carismático aludiendo a aspectos vinculados con su personalidad, decidimos incluir cuatro afirmaciones en forma de ítems que derivan de las conclusiones extraídas de sus ordenaciones. Con esto se pretendía comprobar si las respuestas a las mismas reflejan el modelo defendido a través de las priorizaciones. Los participantes deben mostrar el grado de acuerdo con las mismas haciendo uso de una escala Likert (donde 1 es muy de acuerdo y 4 en desacuerdo).

Los resultados obtenidos muestran un nivel de acuerdo general de los resultados presentados de un $90.69 \%$ (92.77\% bloque factores, $87.7 \%$ competencias y $91.6 \%$ estrategias), por este motivo, se decide cerrar el proceso al estimarse que se ha alcanzado un alto nivel de consenso en las respuestas sobre los objetivos del estudio.

Haciendo referencia a las impresiones de los participantes sobre los ítems relacionados con los resultados obtenidos (Tabla 9), vemos que están de acuerdo en afirmar que para llevar a buen término el proyecto, es imprescindible que el/la emprendedor/a sea carismático/, perseverante y resolutivo/a, contando esta afirmación con una media de 1.4 (desviación típica 0.527 ). Por otro lado, las otras afirmaciones presentadas son puntuadas con valores intermedios, como es el caso de la dedicación de tiempo y esfuerzo en el proyecto de emprendimiento es más relevante que el propio proyecto y su planificación, donde la media de las puntuaciones lo sitúan con un valor de 2.67 (desviación típica 0.866). Con este mismo valor se encuentra la afirmación que hace referencia a que los aspectos relacionados con el proyecto de emprendimiento son 
menos relevantes para el éxito del proyecto que aquellos relacionados con las competencias y valores personales del emprendedor, (media 2.67) (desviación típica 0.707). Lo mismo ocurre con las opiniones acerca de que las características personales influyen directamente en el éxito del proyecto resultando difícil formar para el emprendimiento, afirmación que es situada en puntos centrales contado con una media de 2.56 (desviación típica 0.726). Estas puntuaciones parecen indicar que existe entre los participantes una cierta falta de confianza en que la formación para el emprendimiento pueda resultar un factor significativo en el éxito del mismo.

Tabla 9

Nivel de acuerdo con las conclusiones del estudio

\begin{tabular}{lcc}
\hline \multicolumn{1}{c}{ Afirmaciones sobre las conclusiones del estudio } & Media & DT \\
\hline $\begin{array}{l}\text { Los aspectos relacionados con el proyecto de emprendimiento y } \\
\text { los conocimientos son menos relevantes para el éxito del proyecto } \\
\text { que aquellos aspectos relacionados con las competencias y valores } \\
\text { personales del emprendedor }\end{array}$ & 2.67 & 0.707 \\
$\begin{array}{l}\text { El/la emprendedor/a tiene una serie de características personales que } \\
\text { influyen directamente en el éxito del proyecto por lo que resulta difícil } \\
\text { formar para el emprendimiento }\end{array}$ & 2.56 & 0.726 \\
$\begin{array}{l}\text { La dedicación de tiempo y esfuerzo en el proyecto de emprendimiento } \\
\text { son más relevantes que el propio proyecto y su adecuada planificación }\end{array}$ & 2.67 & 0.866 \\
$\begin{array}{l}\text { Para llevar a buen término el proyecto, es imprescindible que el/la } \\
\text { emprendedor/a sea carismático/a, perseverante y resolutivo/a }\end{array}$ & 1.44 & 0.527 \\
\hline
\end{tabular}

\section{Discusión y conclusiones}

Los resultados del estudio muestran un alto nivel de acuerdo de los emprendedores consolidados en torno a los factores de éxito y las competencias para el desarrollo de su carrera profesional: aspectos personales relacionados con la trayectoria profesional y vital (motivacionales, valores, generación de alianzas y creación de redes de apoyo, inversión de tiempo y esfuerzo) y aspectos vinculados a la planificación empresarial y gestión del proyecto (planificación de la idea de negocio, crear un producto con factor diferenciador, planificación comercial, innovación y cambio). Sin embargo, las aportaciones parecen privilegiar a los que conforman el primer grupo, las características personales. Estos resultados están en consonancia con los hallazgos de las investigaciones de Partida et al. (2012) y Sastre (2013). Por tanto, podemos afirmar que los resultados se identifican con una concepción del emprendimiento basado en el modelo que ofrece la literatura más orientado a las características del emprendedor que a los conocimientos del sector o la formación en emprendimiento. Emprender se relaciona con saber hacer $\mathrm{y}$, por tanto, se considera una competencia a desarrollar que lleva asociada aspectos como el carisma, perseverancia, dedicación de tiempo, esfuerzo, constancia, valores personales o búsqueda de soluciones (Pellicer, 2014). Esta se relaciona con el aprendizaje 
de estrategias comunes a las personas que emprenden independientemente del sector en el que lo hagan y es identificada como factor de éxito principal en el desarrollo y consolidación del proyecto emprendedor. Este aspecto se vincula a los resultados de nuestra investigación donde las competencias más valoradas para propiciar el éxito del proyecto son las comunicativas, de trato personal, las relacionadas con el conocimiento de uno mismo o con la capacidad de resolución de problemas. De esta forma, se deja en un segundo plano la relevancia de la formación en emprendimiento y cobra protagonismo el fomento de la competencia emprendedora y aquellos aspectos vinculados a la misma que se relacionan con la idea del liderazgo emprendedor y carismático, identificado como principal factor de éxito en la consolidación de las ideas de negocio en algunas investigaciones (Van Hemmen et al., 2013; Varga, 2010).

Teniendo en cuenta estas consideraciones, cabe reflexionar sobre qué tipo de iniciativas pueden permitir la adquisición de la competencia emprendedora teniendo en cuenta su relevancia en la puesta en marcha y consolidación de una idea de negocio. En este sentido, una de las opciones a medio plazo sería el fomento del emprendimiento desde las etapas escolares tempranas y su trabajo desde el aula (Cárdenas \& Montoro, 2017; GEM, 2017; LOMCE, 2013). Más a corto plazo, se hace necesario la puesta en práctica de acciones orientadas al fomento de esta competencia y en las que se trabaje el desarrollo personal orientado al emprendimiento a través de aspectos como trabajo en equipo, motivaciones de logro, esfuerzo personal, resolución de problemas, toma de decisiones y liderazgo entre otras, temáticas percibidas como factores de éxito del proyecto emprendedor.

Las limitaciones del estudio se relacionan con el bajo número de participantes, sin embargo, el número final de los mismos se ajusta a las recomendaciones que hace Landeta (2002) para esta metodología. Asimismo, esta investigación se podría completar en el futuro con un estudio más amplio tipo encuesta con mayor representación de emprendedores en diversos sectores, lo que permitiría identificar en una mayor población las competencias emprendedoras relacionadas con el éxito de los proyectos o si estas varían teniendo en cuenta el tipo de empresa o el sector a la que esté dedicada.

\section{Referencias}

Alemany, L., Álvarez, C., Planellas, M., \& Urbano, D. (2011). Libro blanco de la cultura emprendedora en España. Barcelona: Fundación Príncipe de Girona/ESADE.

Bacigalupo, M., Kampylis, P., Punie, Y., \& Van den Brande, G. (2016). EntreComp: The Entrepreneur-ship Competence Framework. Luxembourg: Publication Office of the European Union. doi: http://dx.doi.org/10.2791/593884

Cabero, J., \& Infante, A. (2014). Empleo del método Delphi y su empleo en la investigación en comunicación y educación. EDUTEC, Revista Electrónica de Tecnología Educativa, 48, 1-16. doi: http://dx.doi.org/10.21556/edutec.2014.48.187

Cárdenas, A. R., \& Montoro, E. (2017). Evaluación de un proyecto de educación emprendedora en la ESO. La visión del alumnado. Revista de Investigación Educativa, 35(2), 563-581. doi: http://dx.doi.org/10.6018/rie.35.2.273221

Davidsson, P., Delmar, F., \& Wiklund, J. (2006). Entrepreneurship and the growth of firms. Cheltelham: Elgar. 
Davidsson, P. (2015). Entrepreneurial opportunities and the entrepreneurship nexus: A re-conceptualization. Journal of Business Venturing, 30(5), 674-695. doi: https://doi. org/10.1016/j.jbusvent.2015.01.002

Dalkey, N., \& Helmer, O. (1962). An experimental application of the Delphi method to the use of experts. California: The RAND Corporation. doi: https://doi.org/10.1287/mnsc.9.3.458

Devece, C., Peris-Ortiz, M., \& Rueda-Armengot, C. (2016). Entrepreneurship during economic crisis: success factors and paths to failure. Journal of Business Research, 69 (11), 5366-5360. doi: https://doi.org/10.1016/j.jbusres.2016.04.139

Global Entrepreneurship Monitor (2017). Global Report 2016/2017. Recuperado de http://www.gemconsortium.org/report/49812

Landeta, J. (2002). El método Delphi: una técnica de previsión para la incertidumbre. Barcelona: Ariel.

Leiva, J. (2013). ¿Quién crea MIPYMES en Costa Rica? Tec Empresarial, 7(2), 9-17. doi: http://dx.doi.org/10.18845/te.v7i2.1509

Ley Orgánica 8/2013, de 9 de diciembre, para la mejora de la calidad educativa. Boletín Oficial del Estado, núm. 295, de 10 de diciembre de 2013, pp. 97858-97921. Recuperada de http://www.boe.es/boe/dias/2013/12/10/pdfs/BOE-A-2013-12886.pdf

López Gómez, E. (2018). El método Delphi en la investigación actual en educación: una revista teórica y metodológica. Educación XXI, 21(1), 17-40. doi: https://doi. org/10.5944/educXX1.15536

Messina, M., \& Hochsztain, E. (2015). Factores de éxito de emprendimiento: un estudio exploratorio con base en técnicas de Data Mining. Tec Empresarial, 9(1), 31-40. doi: http://dx.doi.org/10.18845/te.v9i1.2206

Ministerio de Economía, Industria y Competitividad (2017). Cifras PyME. Datos Noviembre 2017: informe español. Madrid: Secretaría general de industria de y la pequeña y mediana empresa. Recuperado de http://www.ipyme.org/Publicaciones/ Cifras_PYME_noviembre2017.pdf

Partida, A., Carrera, M. M., \& Villarreal, L. A. (2012). Análisis de las motivaciones, factores de éxito y obstáculos al espíritu empresarial: estudio comparativo entre México, EEUU y Turquía. Innovaciones de Negocios 9(18), 207-231.

Pellicer, C. (2014). El emprendedor, ¿nace o se hace? Cómo educar el talento emprendedor. Padres y maestros, 355, 6-10.

Porras, J. A., Oliveras, G., \& Vigier, H. P. (2013). Probabilidades de éxito para la creación de empresas: implicaciones sobre la educación emprendedora. Revista FIR, FAEDPYME International Review, 2(4), 42-48.

Red GEM España Observatorio del Emprendimiento (2017). Global entrepreneurship monitor. Informe España, 2016. Santander: Editorial de la Universidad de Cantabria Recuperado de http://www.gem-spain.com/wp-content/uploads/2015/03/InformeGEM2016.pdf

Reguant-Álvarez, M., \& Torrado-Fonseca, M. (2016). El método Delphi. REIRE, Revista d'Innovació i Recerca en Educació, 9(1), 87-102. doi: https://doi.org/10.1344/ reire2016.9.1916

Sastre, R. (2013). La motivación emprendedora y los factores que contribuyen con el éxito del emprendimiento. Revista Ciencias Administrativas. FCE. UNLP, 1(1), 1-10. 
Van Hemmen, S., Urbano, D., \& Álvarez, C. (2013). Charismatic leadership and entrepreneurial activity: an empirical analysis. Innovar, 23(50), 53-66.

Vargas, M. (2010). Emprendimiento y liderazgo. Revista Académica e Institucional, 87, 65-72.

Wang, Y. L., Ellinger, A. D., \& Jim Wu, Y. C. (2013). Entrepreneurial opportunity recognition: An empirical study of R\&D personnel. Management Decision, 51(2), 248-266. doi: https://doi.org/10.1108/00251741311301803

Fecha de recepción: 9 de febrero de 2018.

Fecha de revisión: 2 de marzo de 2018.

Fecha de aceptación: 7 de mayo de 2018. 